\title{
Characterization and applications of auto- locked vacuum-sealed diode lasers for precision metrology
}

Cite as: Rev. Sci. Instrum. 90, 085113 (2019); https://doi.org/10.1063/1.5112760

Submitted: 03 June 2019 . Accepted: 06 August 2019. Published Online: 30 August 2019

H. C. Beica (D), A. Pouliot (D, A. Carew, A. Vorozcovs, N. Afkhami-Jeddi, T. Vacheresse, C. Carlse, P. Dowling, B. Barron, and A. Kumarakrishnan

\section{ARTICLES YOU MAY BE INTERESTED IN}

An open-source high-frequency lock-in amplifier

Review of Scientific Instruments 90, 094701 (2019); https://doi.org/10.1063/1.5083797

Current-feedback-stabilized laser system for quantum simulation experiments using $\mathrm{Yb}$ clock transition at $578 \mathrm{~nm}$

Review of Scientific Instruments 90, 083002 (2019); https://doi.org/10.1063/1.5110037

Determining velocity of detonation using high-resolution time domain reflectometry Review of Scientific Instruments 90, 085005 (2019); https://doi.org/10.1063/1.5100750

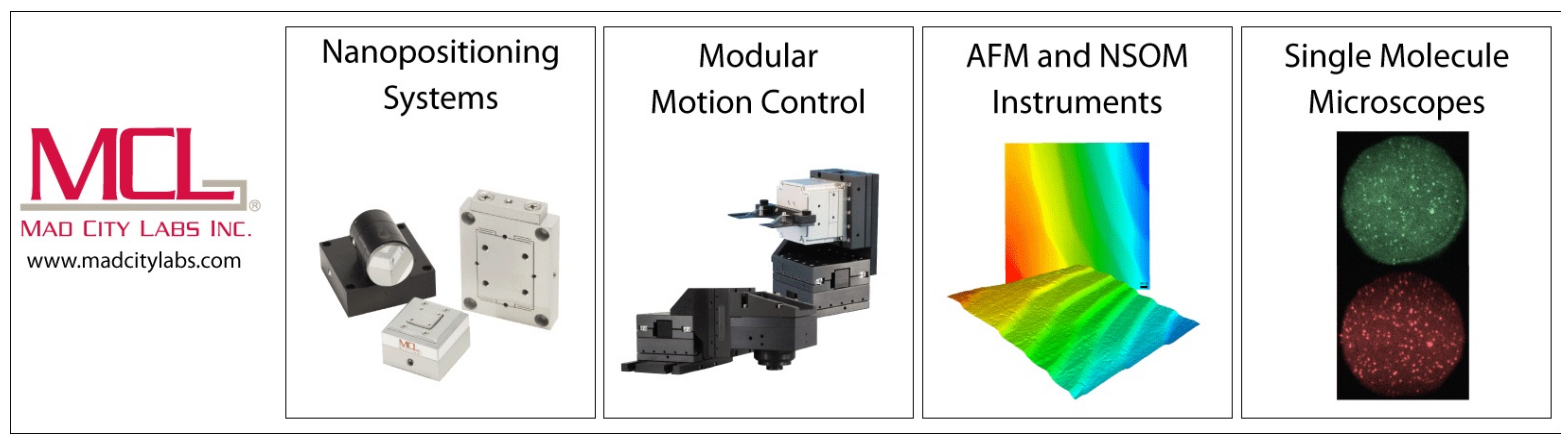




\title{
Characterization and applications of auto-locked vacuum-sealed diode lasers for precision metrology
}

Cite as: Rev. Sci. Instrum. 90, 085113 (2019); doi: 10.1063/1.5112760

Submitted: 3 June 2019 - Accepted: 6 August 2019 •

Published Online: 30 August 2019

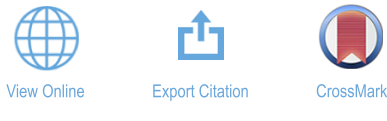

H. C. Beica, (D) A. Pouliot, (D) A. Carew, A. Vorozcovs, N. Afkhami-Jeddi, T. Vacheresse, G. Carlse, P. Dowling, B. Barron, and A. Kumarakrishnan ${ }^{\text {b) }}$

\author{
AFFILIATIONS \\ Department of Physics and Astronomy, York University, 4700 Keele Street, Toronto, Ontario M3J 1P3, Canada \\ a) Electronic mail: hcbeica@gmail.com

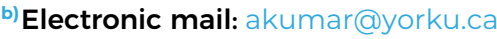

\begin{abstract}
We demonstrate the performance characteristics of a new class of vacuum-sealed, autolocking diode laser systems and their applications to precision metrology. The laser is based on adaptations of a design that uses optical feedback from an interference filter and it includes a vacuum-sealed cavity, an interchangeable base-plate, and an autolocking digital controller. A change of the base-plate allows operation at desired wavelengths in the visible and near infrared spectral range, whereas the autolocking ability allows the laser to be tuned and frequency stabilized with respect to atomic, molecular, and solid-state resonances without human intervention using a variety of control algorithms programmed into the same controller. We characterize the frequency stability of this laser system based on the Allan deviation (ADEV) of the beat note and of the lock signal. We find that the ADEV floor of $2 \times 10^{-12}$ and short-term linewidth of $\sim 200 \mathrm{kHz}$ are strongly influenced by current noise and vacuum sealing. Reducing the current noise and cavity pressure decreases the ADEV floor and increases the averaging time at which the floor occurs, which is a signature of long-term stability. We also show that evacuating the cavity to $\sim 1$ Torr reduces the range of the correction signal of the feedback loop by approximately one order of magnitude, thereby increasing the lock range of the controller. The long-term stability allows the laser to be incorporated into a commercial gravimeter for accurate measurements of gravitational acceleration at the level of a few parts-per-billion, which are comparable to values obtained with an iodine-stabilized He-Ne laser. The autolocking and pattern-matching features of the controller allow the laser to be tuned and stabilized with respect to a temperature tunable transmission spectrum of a fiber-Bragg grating. This capability may be suitable for the development of a differential absorption LIDAR transmitter that can generate data at both on-line and off-line lock points using a single laser.
\end{abstract}

Published under license by AIP Publishing. https://doi.org/10.1063/1.5112760

\section{INTRODUCTION}

There has been wide-spread interest in developing narrowlinewidth laser sources with excellent long-term stability for applications in precision spectroscopy, atomic frequency standards, atom trapping ${ }^{3}$ and atom interferometry, ${ }^{4}$ and precision metrology related to industrial and space applications. ${ }^{5-7}$ Typically, these laser sources are realized by frequency stabilizing laser diodes, distributed feedback (DFB), and distributed Bragg reflector (DBR) lasers to external cavities of varying designs such as compact Littrow cavities, ${ }^{1,8}$ Littman-Metcalf resonators, ${ }^{9,10}$ Fabry-Perot etalons, ${ }^{11,12}$ the internal cavity of the laser diode, ${ }^{13}$ monolithic resonators, ${ }^{14}$ and optical fibers. ${ }^{15}$ Since the frequency stability of these laser systems can often surpass commonly available radio frequency standards such as rubidium vapor cell clocks, ${ }^{16}$ there is also considerable interest in distributing stable optical frequency standards across research laboratories.

In this work, we show that adaptations of a widely used diodelaser design can provide excellent long-term frequency stability for applications such as gravimetry and LIDAR-based remote sensing. In particular, we have adapted an external cavity diode laser (ECDL) design based on interference filter (IF) feedback, developed a digital 
autolocking controller that can stabilize the laser frequency without human intervention, and studied the improvements in stability that can be realized by reducing current noise and by vacuum-sealing the cavity. Well known designs have used gratings, etalons, and beam splitters to provide optical feedback to the laser diode. ${ }^{18-25}$ Representative studies focused on improving the short-term stability are described in Refs. 23, 26, and 27. In the widely used Littrow design, scanning the laser frequency with a piezomounted grating changes the optical feedback as well as the length of the external cavity. This disadvantage can be overcome by positioning the grating at a carefully chosen pivot point, ${ }^{23}$ whereas the overall stability of the Littrow cavity can be improved using a monolithic design, hermetic sealing, back-filling with inert gases, and improving vibration isolation. ${ }^{25}$ The work in Ref. 23 showed the mode hop-free tuning range of a modified Littrow design in the 780-nm band that had reduced sensitivity to acoustic noise. The mode hopfree tuning range of these systems can be significantly extended by synchronous scanning of the current and the grating using a feed-forward circuit. In comparison, IF-stabilized diode lasers have the advantage that the filter used for wavelength selection is separate from the piezomounted mirror which changes the length of the laser cavity. This design also reduces distortions of the spatial beam profile. These two effects make it potentially easier to achieve mode hop-free tuning over the free spectral range of the cavity.

Previous work ${ }^{21,22}$ showed that the IF design exhibited a narrow linewidth (tens of $\mathrm{kHz}$ ) and a tuning range of several nanometers achieved by changing the incidence angle of the filter. In our work, we demonstrate the operation of IF lasers at both $633 \mathrm{~nm}$ and $780 \mathrm{~nm}$ using the same vacuum-compatible laser head design and an autolock controller. The desired operating wavelength is achieved by changing the optical elements and laser diode. In ECDL designs that have been commercialized, the stability of the laser is achieved by temperature and current control of the laser diode and temperature stabilization of the enclosure containing the laser head. However, long-term ambient temperature and pressure drifts necessitate a correction signal with a large range, thus limiting the time over which the laser frequency can remain actively stabilized (locked). Our design has addressed this limitation by allowing the laser cavity to be pumped out. ${ }^{28-30}$ To bring the laser cavity back into alignment after pump-out, we use a vacuumcompatible feedthrough to realign the angle of the IF from outside the cavity to compensate for the change in the refractive index.

Our results indicate that the suppression of pressure-induced frequency drifts results in a nearly ten-fold reduction of the correction signal amplitude, thereby increasing the lock duration and improving long-term stability. We show that both a reduction in the current noise and a reduction in the pressure inside the laser cavity result in a reduced overall Allan deviation (ADEV) and an increase in the averaging time at which the floor of the ADEV occurs, thereby indicating improved long-term stability. The autolocking feature facilitates locking and tuning with respect to atomic, molecular, and solid-state references. The advantages of autolocking coupled with the long-term stability allow the laser system to be integrated with an industrial gravimeter to achieve accurate measurements of gravitational acceleration at the level of a few parts-per-billion (ppb). Furthermore, we show that the laser can be frequency stabilized with respect to a temperature tunable transmission peak from a fiberBragg grating (FBG). Since a pattern-matching algorithm can be used for autolocking, this demonstration allows the laser system to serve as the basis for a differential absorption (DIAL)-type LIDAR transmitter that can be operated at both on-line and off-line lock points.

\section{EXPERIMENTAL DETAILS}

Figure 1(a) shows a schematic of the laser head. The collimated output of the laser diode passes through a variable beam splitter, which transmits a small fraction of the laser power through the IF with a $0.5-\mathrm{nm}$ FWHM passband. The frequency band selected by the IF is optically fed back into the diode by a "cat's eye" retroreflector consisting of a piezomounted mirror placed at the focal length of a lens. ${ }^{21,22}$ The external cavity length, measured from the laser (a)

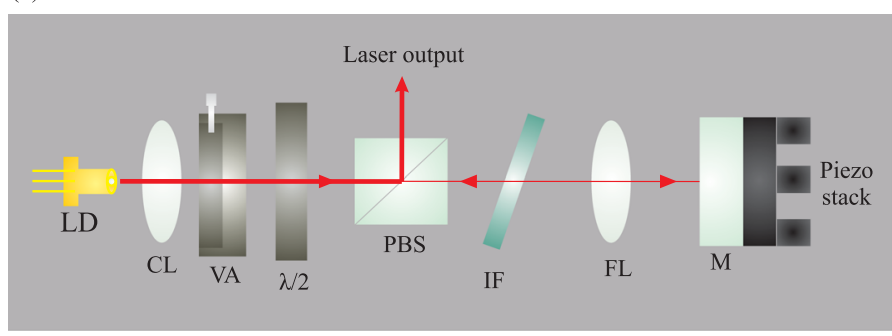

(b)

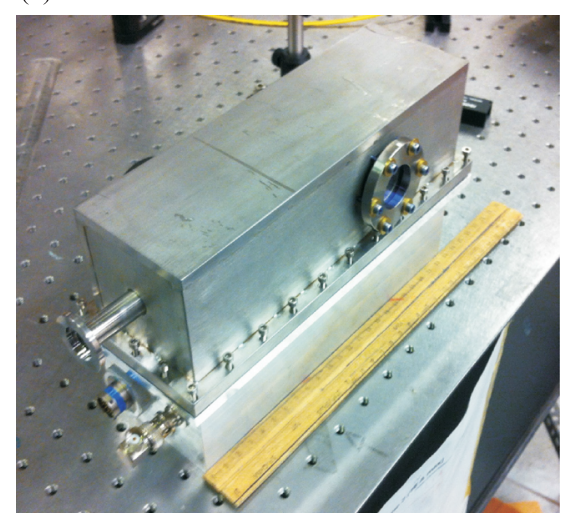

FIG. 1. (a) Schematic of the IF laser design. The optical elements are LD—laser diode, CL—collimating lens, VA-variable aperture, $\lambda / 2-$ half wave-plate, PBS-polarizing beam splitter, IF-interference filter, FL-focusing lens, and $\mathrm{M}-$ mirror. (b) Photograph showing pump-out port, O-ring seals of the window and enclosure, and electrical feedthroughs in the base. 
diode to the retroreflecting mirror, is $10 \mathrm{~cm}$. The output of the laser cavity $(50-100 \mathrm{~mW})$ is the vertically polarized reflection from the variable beam splitter. All the optical elements of the laser cavity are mounted on a compact, temperature-stabilized aluminum baseplate (controlled to $\pm 1 \mathrm{mK}$ ). The base-plate and the diode can be easily interchanged to allow the laser to operate within a selected wavelength range. A variable aperture mounted near the laser diode is used to spatially filter the feedback light. ${ }^{29,30}$ The angle of the IF can be adjusted from outside the laser cavity using a vacuum feedthrough connected to an assembly with a gear ratio of either 18:1 (cavity type A) or 100:1 (cavity type B). Cavity type B is also designed with screw holes that have escape channels for trapped gases. An electrical vacuum feedthrough also enables the laser set-points (current, temperature, and piezovoltage) to be controlled from outside the laser cavity, and a built-in pressure sensor allows the pressure inside the cavity to be monitored. The base-plate is covered with a stainless-steel or aluminum enclosure with an O-ring for vacuum sealing. The enclosure also has a welded pump-out port for evacuating the cavity and an O-ring-sealed view-port for the laser output, as shown in Fig. 1(b).

The autolock controller consists of a low-cost, single-board computer (SBC) with analog-to-digital (A/D) and digital-to-analog (D/A) capability that acts as a specialized lock-in amplifier. It is also responsible for unattended start-up of the laser and laser mode monitoring functions. In order to tune the laser to the desired spectral location (frequency) from a cold start condition, the controller performs pattern matching between Doppler-free peaks obtained by scanning the laser frequency and reference peaks stored in the processor's memory. The incoming spectral signals are compared with the reference waveforms using a sliding correlation algorithm, which determines the control voltages required for adjusting the laser frequency to the desired lock point. The system has a scan amplitude of less than $1 \mathrm{MHz}$ when locked, and it can relock for frequency drifts up to $10 \mathrm{GHz}$ without human intervention. The controller is also equipped with an autocalibration module, which allows a variety of laser operating parameter combinations to be systematically explored in order to determine optimal settings required to avoid operating the laser diode in a multimode condition or near a mode hop. The controller builds and stores a library of settings for a particular laser diode and periodically recalibrates the determined parameters to adjust for aging of the laser diode to ensure stable operation.
The SBC is equipped with two analog-to-digital conversion (ADC) input pipelines, a high-speed input stage, and a backgroundmonitoring stage. The high-speed input stage samples one photodiode signal and a copy of the piezosignal (for definitive referencing and synchronization) simultaneously at a $100-\mathrm{kHz}$ sampling rate with 16-bit resolution. The background monitoring stage operates at a $120 \mathrm{~Hz}$ sampling rate and is used for recording operating conditions of the laser such as current, temperature, and pressure. The $\mathrm{SBC}$ is also equipped with two digital-to-analog converter (DAC) output stages. The first stage operates at $48 \mathrm{kHz}$ and 16-bit resolution and is used to synthesize triangular and sinusoidal waveforms for lookup scans and lock-in operation. The second DAC stage is used to generate slow-varying control signals and set-points for the laser controller. The output for the piezo is fed into a high-voltage amplifier to enable a large $(\sim 10 \mathrm{GHz})$ scan range.

An analog preamplifier brings the input analog signals within the analog input range of the ADC. After the signals are preamplified and digitized, the on-board software performs the appropriate processing functions (laser start-up, linear scan, or continuous lock-in operation). The output parameters are fed out to the DACs and the corrections are immediately applied to the laser current and piezo. The SBC is equipped with $128 \mathrm{MB}$ of RAM and runs at a $500-\mathrm{MHz}$ clock rate. These parameters allow for complex digital signal processing operations to be performed quickly, such as sliding correlation on large $(\mathrm{N}=1024)$ samples and longterm finite impulse response filtering. The SBC is also equipped with on-board nonvolatile (flash) storage that is used to log operating data for subsequent review. The SBC controller can be operated locally, via a $4.3^{\prime \prime}$ touch screen mounted on the case or remotely via a TCP/IP connection using Ethernet through a LabVIEW interface.

Our laser characterization shown in Fig. 2 also involves a vibration isolation platform with a resonant frequency of $\sim 1 \mathrm{~Hz}$. Two identical diode-laser systems are mounted on this platform and locked to neighboring resonances in iodine or rubidium vapor to produce beat notes ranging from 10 to $90 \mathrm{MHz}$. The short-term laser linewidth is inferred from a Fourier transform of the beat note acquired in $50 \mathrm{~ms}$ using a spectrum analyzer with a resolution of $1 \mathrm{~Hz}$ and scan range of $9 \mathrm{kHz}-26.5 \mathrm{GHz}$. The long-term frequency stability is inferred from the ADEV of both the beat note and the lock signal. These data sets are of the form $y_{i}(\tau), y_{i+1}(2 \tau), \ldots$, where $\tau$ is the sampling time. We then calculate an overlapping ADEV

Saturated absorption signal detection

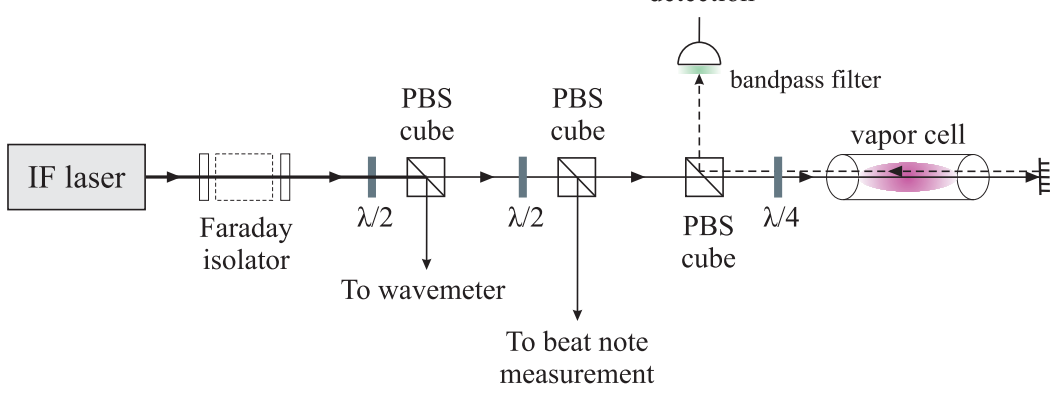

FIG. 2. Setup for laser diagnostics, which was mounted on a vibration isolation platform. The saturated absorption spectrometer involves a compact dual-pass configuration. 
based on ${ }^{31}$

$$
\sigma(\tau)=\sqrt{\sum_{i=1}^{N-1} \frac{1}{2(N-1)}\left(y_{i+1}-y_{i}\right)^{2}} .
$$

The lock signal data is obtained by stabilizing the laser frequency with respect to a single hyperfine resonance in a compact saturated absorption spectroscopy setup in which the pump beam is retroreflected through the vapor cell to generate a weak counterpropagating probe beam. ${ }^{32}$ The beat note is recorded on a Si-PIN photodiode with a rise time of $1 \mathrm{~ns}$, whereas the lock signal data is recorded with a $10-n s$ rise time photodiode. A wavemeter with a resolution of $50 \mathrm{MHz}$ is used to coarse tune the laser frequency. The calibration of the frequency offsets and drifts is obtained from measured hyperfine splittings such as those in Ref. 33 and from wavemeter readings. For lock signal analysis, a data card with a sampling rate of 48000 samples/s is used, whereas for the beat note analysis, we use a data card with a sampling rate of $1 \mathrm{GS} / \mathrm{s}$. A separate $\mathrm{C}++$ program is used to determine the frequency of the beat note based on counting the zero crossings.

For the iodine data, the frequency stabilization is accomplished using the third derivative of the saturated absorption signal since the resonance widths are relatively narrow $(\sim 1 \mathrm{MHz})$ and superposed on a large Doppler background. In rubidium, stabilizing the laser frequency using pattern matching was successful since the shapes of the resonances are reliably known without repeating molecular series.
Alternatively, the laser frequency can be stabilized using first derivative feedback. The aforementioned technique is also used to lock the laser frequency to a FBG transmission peak. The time constant of the feedback loop is $\sim 1 \mathrm{~s}$ for the iodine-stabilized laser systems and $\sim 50 \mathrm{~ms}$ for the rubidium and FBG systems. Both the error signal that is fed back to the laser cavity and the correction signal are monitored by the controller. The correction signal represents the drift of the accumulated error signal over the lock duration, namely, the voltage excursions made by the error signal during the lock period. These voltages serve as the inputs for the feedback correction values. Each voltage correction is added to the preceding value, until the full voltage range of the controller is reached. These voltages are converted to frequencies through a calibration.

For the data associated with the vacuum-sealed lasers, the laser cavity is evacuated using a roughing pump and a $2-\mathrm{m}$ long bellows hose. Prior to recording lock data, the vacuum enclosure of the laser is valved off and the bellows disconnected. The pressure of the sealed laser cavity is monitored using a Pirani gauge. At each pressure setting, the optical feedback is adjusted from outside the cavity by tuning the IF angle.

\section{RESULTS AND DISCUSSION}

\section{A. Laser characterization}

Figure 3(a) shows the Doppler-broadened resonance in a $10-\mathrm{cm}$ long iodine vapor cell. The observed hyperfine resonances can
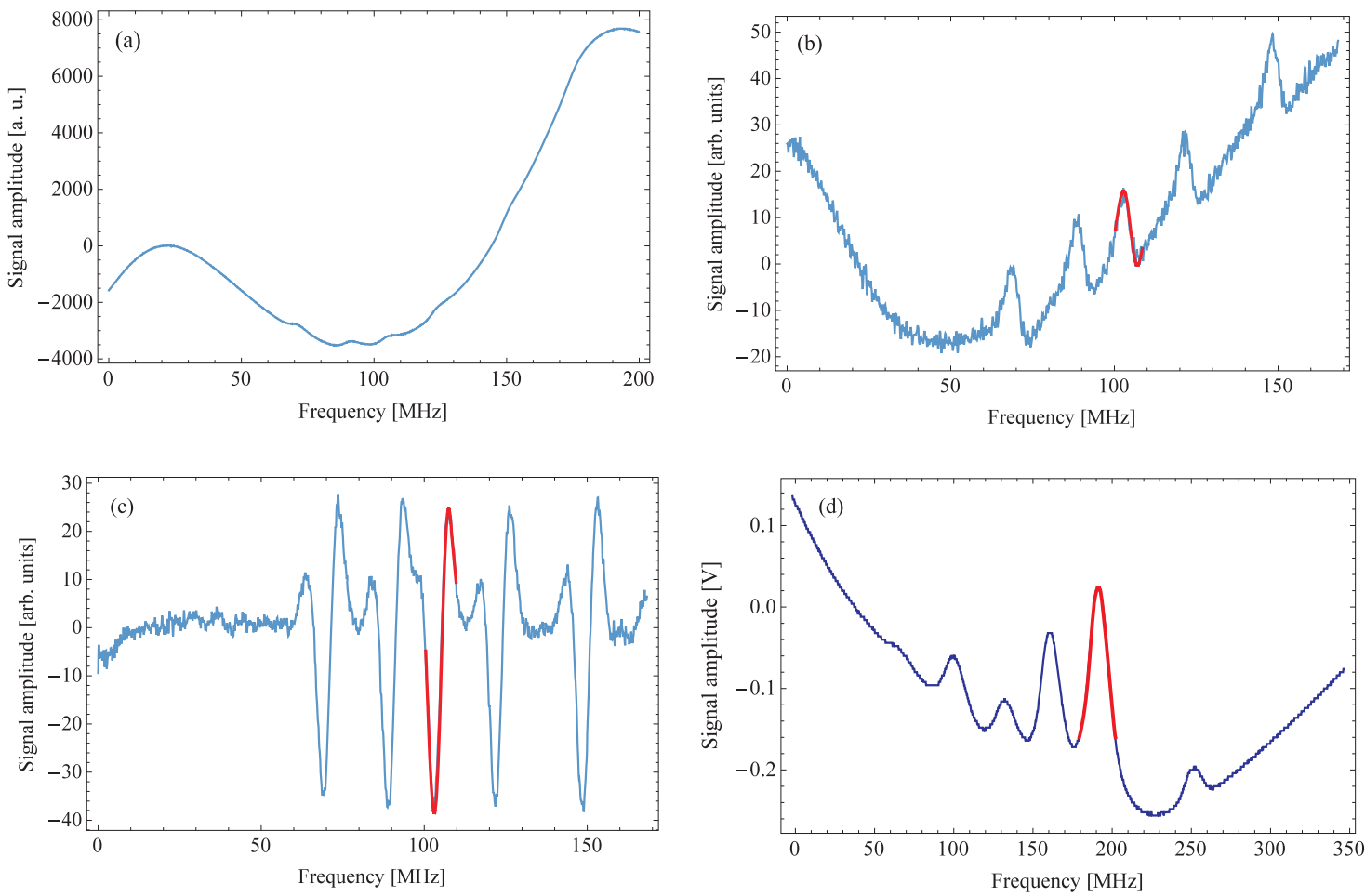

FIG. 3. Representative saturated absorption spectra showing typical lock points highlighted in red. (a) $P(33) 6-3$ Doppler-broadened resonance in iodine vapor. (b) First derivative of the $P(33) 6-3$ Doppler-broadened resonance. The FWHM inferred from the fit is $(5.1 \pm 0.1) \mathrm{MHz}$. (c) Third derivative of the $\mathrm{P}(33) 6-3 \mathrm{spectrum}$. The FWHM inferred from the fit is $(2.9 \pm 0.1) \mathrm{MHz}$. (d) Hyperfine spectrum of the $F=3 \rightarrow F^{\prime}=2,3,4$ transition in ${ }^{85} \mathrm{Rb}$ vapor. The FWHM inferred from the fit is (12.9 \pm 0.2$) \mathrm{MHz}$. 
be clearly discriminated using a first derivative error signal, as shown in Fig. 3(b). Since the resonances are relatively narrow, the tightest locks were achieved using third derivative spectroscopy, as shown in Fig. 3(c). Another advantage of this method is that it removes the Doppler background. Figure 3(d) shows representative hyperfine spectra in ${ }^{85} \mathrm{Rb}$ which are relatively broad $(\sim 10 \mathrm{MHz})$ due to power broadening.

Figure 4(a) shows the lock signal and correction signal for a $780-\mathrm{nm}$ laser system (design type A) that is frequency stabilized at atmospheric pressure using first-derivative spectroscopy and a current controller with a characteristic power spectral density of $200 \mathrm{nA} / \sqrt{\mathrm{Hz}}$. The figure shows that the lock signal is centered about the lock point with excursions of $\sim 1 \mathrm{MHz}$. In contrast, the correction signal shows a drift of several tens of megahertz. Figure 4(b) shows the same data with the laser cavity evacuated to a pressure of 1.6 Torr. Although, the lock signal excursions are about the same, the overall range of the correction signal has been reduced by nearly a factor of 7 . Similar reduced ranges for the correction signal were observed using third derivative spectroscopy in iodine and pattern matching algorithms in rubidium. ${ }^{29}$ Additionally, the correction signals at atmospheric pressure are strongly correlated with pressure variations, whereas the corresponding correction signals at 1.6 Torr are uncorrelated. The features of the correction signals for the pressure-sealed lasers indicate an obvious advantage, namely, an extended lock duration and potentially greater long-term stability. This aspect is unambiguously shown in studies of the ADEV that follow. However, since the ADEV is significantly affected by both laser current noise and cavity pressure, we first present studies of the effects of current noise.

Figure 5(a) shows the ADEV of the beat note at atmospheric pressure for a 780-nm cavity of design type A. A fit to the curve suggests that the data is dominated by white noise at short times, and the floor reaches a value of $2.5 \times 10^{-11}$ at $\tau=40 \mathrm{~s}$. Figure 5(b) shows the corresponding ADEV curve for the lock signal. Here, the floor reaches a value of $2 \times 10^{-11}$ at $\tau=80 \mathrm{~s}$. Figures. 5(c) and 5(d) show the ADEVs of the beat note and lock signal for a cavity of design type A using a current controller with a much smaller power spectral density $(<100 \mathrm{pA} / \sqrt{\mathrm{Hz}})$, which is based on the design in Ref. 34 . Figure 5(c) shows that the ADEV of the beat note is reduced by nearly an order of magnitude, and that the floor value is $2 \times 10^{-12}$ at an extended averaging time of $\tau=300 \mathrm{~s}$. Similarly, in Fig. 5(d), the ADEV of the lock signal has a floor of $1.5 \times 10^{-12}$ for $\tau=300 \mathrm{~s}$. Based on these data sets, it is evident that current noise strongly impacts the ADEV and improves the long-term stability, thereby extending the lock duration. Under these conditions, the floor of the ADEV of the laser intensity, which is also influenced by current noise, is $5 \times 10^{-6}$ at $\tau=10 \mathrm{~s}$. We have also displayed polynomial fits to the data in Figs. 5(a) and 5(c). Here, the negative coefficients are attributed to the dominance of the $\tau^{-1 / 2}$ terms.

Current noise also influences the laser linewidth, as shown by the data in Figs. 6(a) and 6(b). In Fig. 6(a), the Fourier transform of the beat note of two similar 780-nm lasers gives an individual laser linewidth of $\sim 1 \mathrm{MHz}$. The linewidth is reduced to $\sim 200 \mathrm{kHz}$ with the lower noise current controller, as shown in Fig. 6(b). The laser linewidth is best modeled by a Voigt profile ${ }^{13}$ with a Gaussian component representing the effect of current noise and a Lorentzian component representing the cavity linewidth. In Ref. 21, the record length of the spectrum analyzer within the scan range permits these components to be resolved. Since our record length does not have sufficient point density, we perform fits to both Gaussian and Lorentzian functions. In both data sets, we find that the laser linewidth data is better represented by a Gaussian fit than a Lorentzian function, suggesting that current noise dominates the effect of the intrinsic cavity linewidth. Although both line shape functions model the central portion of the curve, the Lorentzian fit misses points in the wings. Accordingly, we find that the fit error for the Gaussian is consistently better than that of the Lorentzian.

In general, the beat note ADEV is considered to be a more representative measure of laser performance. In comparison, the lock signal ADEV can potentially be influenced by drifts in the lock point due to other external factors such as magnetic fields. However, based on the data in Fig. 5, it is evident that both measures are qualitatively similar. Since our lock studies extend to only about $1 \mathrm{~h}$-in which perturbations of the lock point are insignificant-we present only ADEVs of the lock signal to show the effects of pressure variations. Relying on the ADEV of the lock signal rather than the ADEV of the beat note also avoids the need to maintain two cavities at identical pressures.
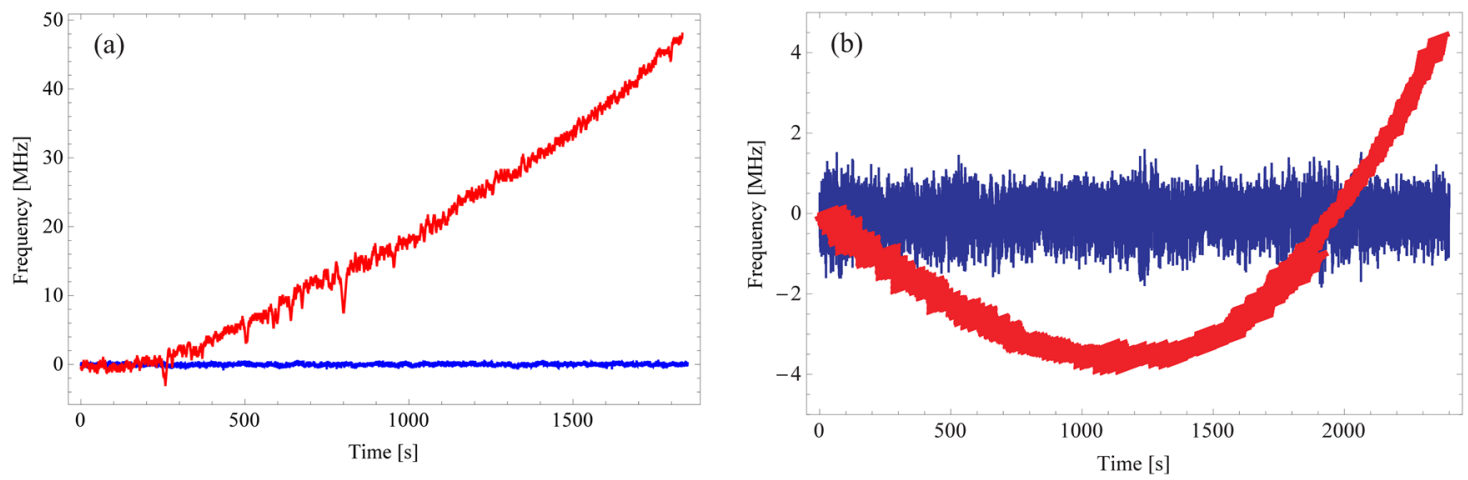

FIG. 4. (a) Lock signal (blue) and correction (red) signal recorded with the laser cavity at atmospheric pressure. (b) Lock signal (blue) and correction (red) signal recorded with the laser cavity evacuated to 1.6 Torr. 

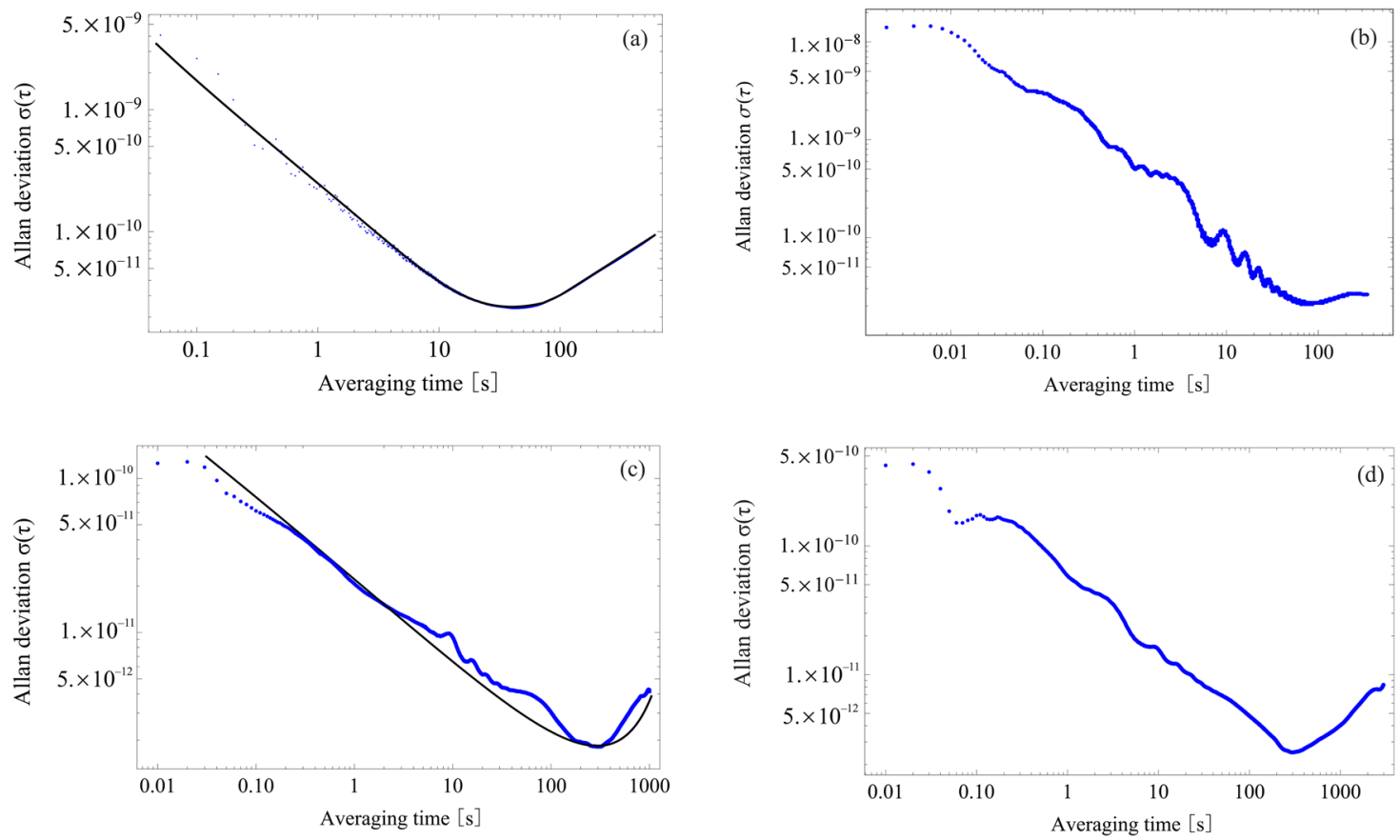

FIG. 5. (a) ADEV of the beat note obtained with two 780-nm lasers of design type A recorded using a current controller with a power spectral density of $200 \mathrm{nA} / \sqrt{\mathrm{Hz}}$. The fit function is $(-2.77 \pm 0.02) \times 10^{-11} \tau^{0}+(1.78 \pm 0.04) \times 10^{-11} \tau^{-1}+(1.453 \pm 0.006) \times 10^{-10} \tau^{-0.5}+(4.06 \pm 0.02) \times 10^{-12} \tau^{0.5}+(2.36 \pm 0.05) \times 10^{-14} \tau$. (b) ADEV of the lock signal of a single laser recorded with a current controller with a power spectral density of $200 \mathrm{nA} / \sqrt{\mathrm{Hz}}$. The ripples in the ADEV curve are attributed to the resonant frequency of the vibration isolation platform. (c) ADEV of the beat note obtained with two 780-nm lasers of design type A recorded using a current controller with a power spectral density of $100 \mathrm{pA} / \sqrt{\mathrm{Hz}}$. The fit function is $(-4.52 \pm 0.04) \times 10^{-11} \tau^{0}+(3.57 \pm 0.06) \times 10^{-11} \tau^{-1}+(4.74 \pm 0.02) \times 10^{-10} \tau^{-0.5}+(7.14 \pm 0.05) \times 10^{-12} \tau^{0.5}+(2.16$ $\pm 0.06) \times 10^{-14} \tau$. (d) ADEV of the lock signal of a single laser recorded with a current controller with a power spectral density of $100 \mathrm{pA} / \sqrt{\mathrm{Hz}}$.

We now examine the effect of pressure variations on the ADEV curves carried out with a 780-nm laser cavity of design type B using a current controller with a power spectral density of $200 \mathrm{nA} / \sqrt{\mathrm{Hz}}$. For this study, we acquire ADEV curves over a single 12-h run to best track relative changes. Based on Fig. 7(a), it is evident that the floor of the ADEV values of the lock signal is reduced by about a factor of
2 at a pressure of 20 Torr compared to its atmospheric value. However, the ADEV increases slightly from 20 Torr to 1 Torr. Although cavity type B had pressure-release holes, both cavity types A and B can be affected by the slow escape of trapped gasses. The epoxy used inside these cavities (part number 353NDPK from Thorlabs) has an outgassing level of $5 \times 10^{-5}$ Torr. We therefore attribute the increase
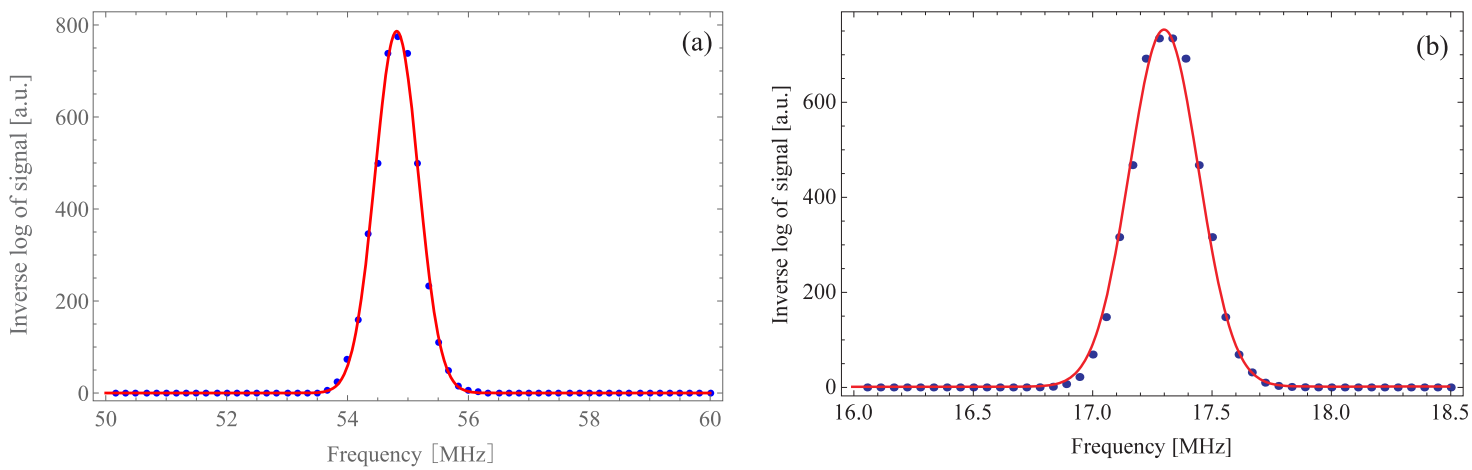

FIG. 6. (a) Short-term beat note of two type-A $780-n m$ laser cavities recorded with a spectrum analyzer and a current controller with a power spectral density of $200 \mathrm{nA} / \sqrt{\mathrm{Hz}}$. The Fourier transform of the beat note is displayed on a linear scale by taking the inverse logarithm. A Gaussian fit gives a FWHM of (1.136 \pm 0.005$) \mathrm{MHz}$. (b) A similar linewidth measurement as in (a), obtained using a current controller with a power spectral density of $100 \mathrm{pA} / \sqrt{\mathrm{Hz}}$. Here, a Gaussian fit gives a FWHM of (221 \pm 1$) \mathrm{kHz}$. 

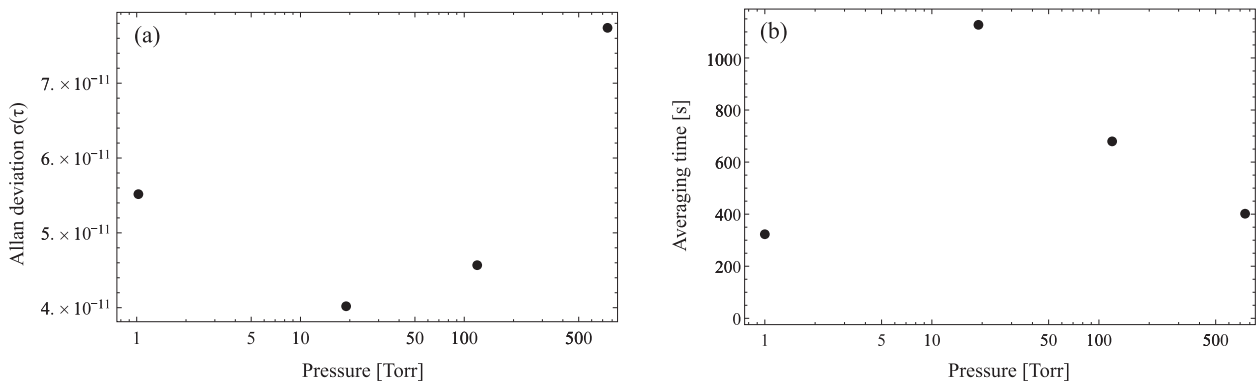

FIG. 7. (a) Floor of ADEV curves of the lock signal as a function of cavity pressure recorded with a 780-nm laser cavity of design type B. (b) Averaging time $\tau$ corresponding to the floor of the ADEV curves in (a) as a function of the pressure inside the laser cavity. below 20 Torr to the escape of trapped gasses inside the laser cavity. The data in Fig. 7(b) shows that pressure reduction extends the value of the averaging time of the ADEV curve by approximately a factor of 3. It is therefore evident that both cavity pressure and current noise can have significant effects on the long-term stability. We find that the averaging time reduces when the pressure is decreased from 20 Torr to 1 Torr, which is consistent with the trends observed in (a). Although pressure variations have a lesser impact on the floor of the ADEV curves than current noise, the extension of the averaging time at reduced pressures is just as pronounced as the effect of reducing the current noise. Therefore, these data illustrate the benefit of using vacuum-sealed cavities for diode-laser systems.

\section{B. Applications to metrology}

In this subsection, we show that the long-term stability and autolocking features of the laser system can be used for precise measurements of gravitational acceleration by integrating the laser system with an industrial gravimeter. Gravimeters are sensitive to variations in the value of the acceleration due to gravity $g$ on the Earth's surface. They can be designed to measure the absolute value of $\boldsymbol{g}$ or relative changes due to temporal effects such as tides and positional variations due to changes in density. Therefore, gravimeters play a ubiquitous role in the exploration of natural resources by detecting characteristic density profiles associated with minerals, petroleum, and natural gas. In particular, these devices provide a noninvasive technique for exploration in wide-area (air, sea, or submersible) mineral assays and borehole mapping for verifying properties of rocks, determination of bulk density detection of cavities, and tidal forecasts. They are also used for seismic monitoring of environmentally sensitive areas that are designated for resource extraction.

The most accurate portable absolute gravimeter is based on a falling corner cube Mach-Zehnder optical interferometer (Scintrex FG5X), ${ }^{5}$ in which one arm containing a retroreflecting corner cube falls in gravity inside a vacuum chamber. Measurements of $\boldsymbol{g}$ accurate to $\sim 2 \mathrm{ppb}$ have been realized by recording the chirped accumulation of fringes over a drop height of $\sim 0.3 \mathrm{~m}$. The accuracy depends on the frequency stability of an iodine-stabilized 633-nm $\mathrm{He}-\mathrm{Ne}$ laser used for interferometry. This laser has a power output of $\sim 100 \mu \mathrm{W}$, a linewidth of $\sim 10 \mathrm{kHz}$, and an ADEV of $\sim 10^{-11}$ that is traceable to a primary frequency standard. In this section, we compare measurements of $\boldsymbol{g}$ under laboratory conditions using an auto-locked 633-nm diode-laser system and an iodine-stabilized $\mathrm{He}-\mathrm{Ne}$ laser.
For the gravimetric measurement, light from the laser source is split into two paths-a reference beam along the horizontal, and a measurement beam along the vertical, which is incident on the corner cube retroreflector. After the corner cube falls in gravity, it is gently brought to rest and reset. The change in path difference between the two arms of the interferometer produces a chirped heterodyne beat note resulting in a signal with zero-crossings which are timed and counted. The value of $\boldsymbol{g}$ is determined from a fit to the resulting displacement-time graph. The precision depends on the drop height, and scales as the square of the drop time. The fit includes corrections for systematic effects such as the Earth's gravitational gradient and laser modulation frequency. Models for tidal variations are also used to correct temporal changes in $\boldsymbol{g}$ so that a baseline of gravity values obtained over a few hours can be used to compare the two laser systems. In this work, the value of $\boldsymbol{g}$ was obtained by performing 100 drops of the corner cube over a period of $5 \mathrm{~min}$, and repeating this sequence every half an hour for several hours.

Figure 8 shows measurements of $\boldsymbol{g}$ using the autolocked diodelaser system stabilized with respect to the $\mathrm{P}(33) 6-3$ branch in iodine vapor at $633 \mathrm{~nm}$ using a current controller with a power spectral density of $200 \mathrm{nA} / \sqrt{\mathrm{Hz}}$. For this data, the maximum power output of the autolocked laser system $(\sim 50 \mathrm{~mW})$ was attenuated to $100 \mu \mathrm{W}$ to match the maximum response of the gravimeter detector. Each individual point in Fig. 8 represents an average of 100 drops of the corner cube. The zero baseline represents the values established by the iodine-stabilized He-Ne laser. The typical standard deviation for a single set of 100 measurements is $18 \mathrm{ppb}$ which corresponds to $18 \mu \mathrm{Gal}$ (where $1 \mathrm{Gal}=1 \mathrm{~cm} / \mathrm{s}^{2}$ ) and the root-mean-square value of the fit residuals of the displacement-time graphs is $3 \mathrm{~nm}$. The overall uncertainty for the entire data set (statistical uncertainty of $3 \mu \mathrm{Gal}$ and a systematic uncertainty of $11 \mu \mathrm{Gal}$ ) shows that the two lasers produce measurements in agreement with each other. The signalto-noise ratio was limited by the input optical power of the detector, which was optimized for the $100-\mu \mathrm{W}$ output of the He-Ne laser. As a result, we can expect further improvements since the autolocked diode-laser system is capable of power outputs of $\sim 100 \mathrm{~mW}$.

We now describe an application of the laser system relevant to LIDAR transmitters. LIDAR sources are widely used for atmospheric pollution monitoring and there has been considerable interest in developing diode laser-based LIDAR sources. ${ }^{35,36}$ The ability of LIDAR to detect water vapor has also enabled these systems to be adapted for planetary exploration such as the investigation of the hydrological cycle on Mars as part of the NASA Phoenix mission. ${ }^{37}$ The autolocking feature of the diode lasers described in this 


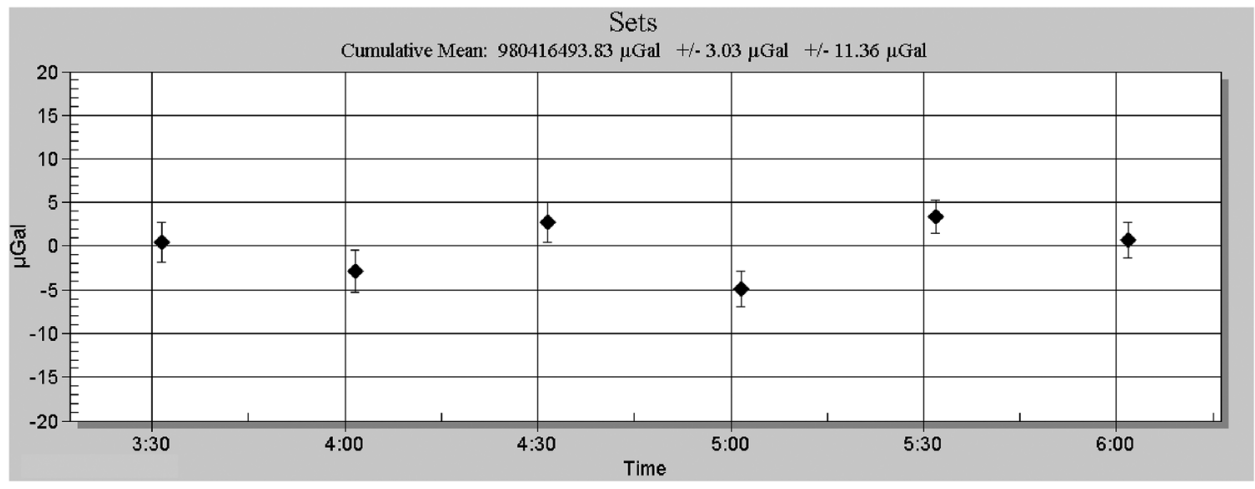

FIG. 8. Measurements of $g$ obtained with an autolocked diode laser at $633 \mathrm{~nm}$ integrated with the Scintrex FG5X gravimeter. Each point represents an average of 100 drops of the corner-cube reflector. This sequence was repeated at intervals of $30 \mathrm{~min}$ for a time period of $3 \mathrm{~h}$. The zero represents the baseline established by an iodine-stabilized $\mathrm{He}-\mathrm{Ne}$ laser.

work coupled with a pattern-matching algorithm allows the laser frequency to be locked to a spectral line and to be tuned with a programmable offset with respect to the peak. In this section, we utilize these features to demonstrate a key step toward the realization of a differential absorption LIDAR (DIAL) transmitter. The standard measurement technique for DIAL-type LIDAR $^{36,38,39}$ involves the absorption and scattering of pulsed laser light propagating through the atmosphere. Light is emitted into the atmosphere, and the light that is scattered back is detected and recorded as a function of propagation time. The density of a desired aerosol can be determined by comparing the intensity of laser backscatter to a reference signal at an off-line wavelength that is not absorbed. A pulsed laser system that can operate at both wavelengths can be realized by integrating the autolocked diode laser with a semiconductor tapered amplifier waveguide ${ }^{40}$ for power amplification and an acousto-optic switch to realize a pulsing module with repetition rates of up to $1 \mathrm{MHz} .{ }^{41}$ Unlike traditional DIAL-type LIDAR transmitters that rely on a pulsed YAG or diode laser and a separate laser-based measurement of the off-line signal, we anticipate using a single autolocked master oscillator that can be locked and tuned to multiple frequencies with respect to atomic or molecular spectral lines as well as their corresponding off-line points. Here, we show that a solidstate temperature-tunable spectral marker (transmission spectrum of FBG) can be calibrated against known hyperfine splittings of atomic lines so that the frequency of a LIDAR transmitter can potentially be extended to a region in which convenient frequency markers are unavailable.

Figure 9(a) shows the transmission peak from an FBG in the $780-\mathrm{nm}$ band, which has a FWHM of $70 \mathrm{MHz}$. The portion of the fiber containing the FBG $(\sim 1 \mathrm{~cm}$ in length) was encased in a thermallyconductive paste and temperature tuned and stabilized with a Peltier cooler so that the transmission peak could be calibrated against rubidium hyperfine lines. Figure 9(b) shows the tunability of the FBG peak as a function of temperature. These data
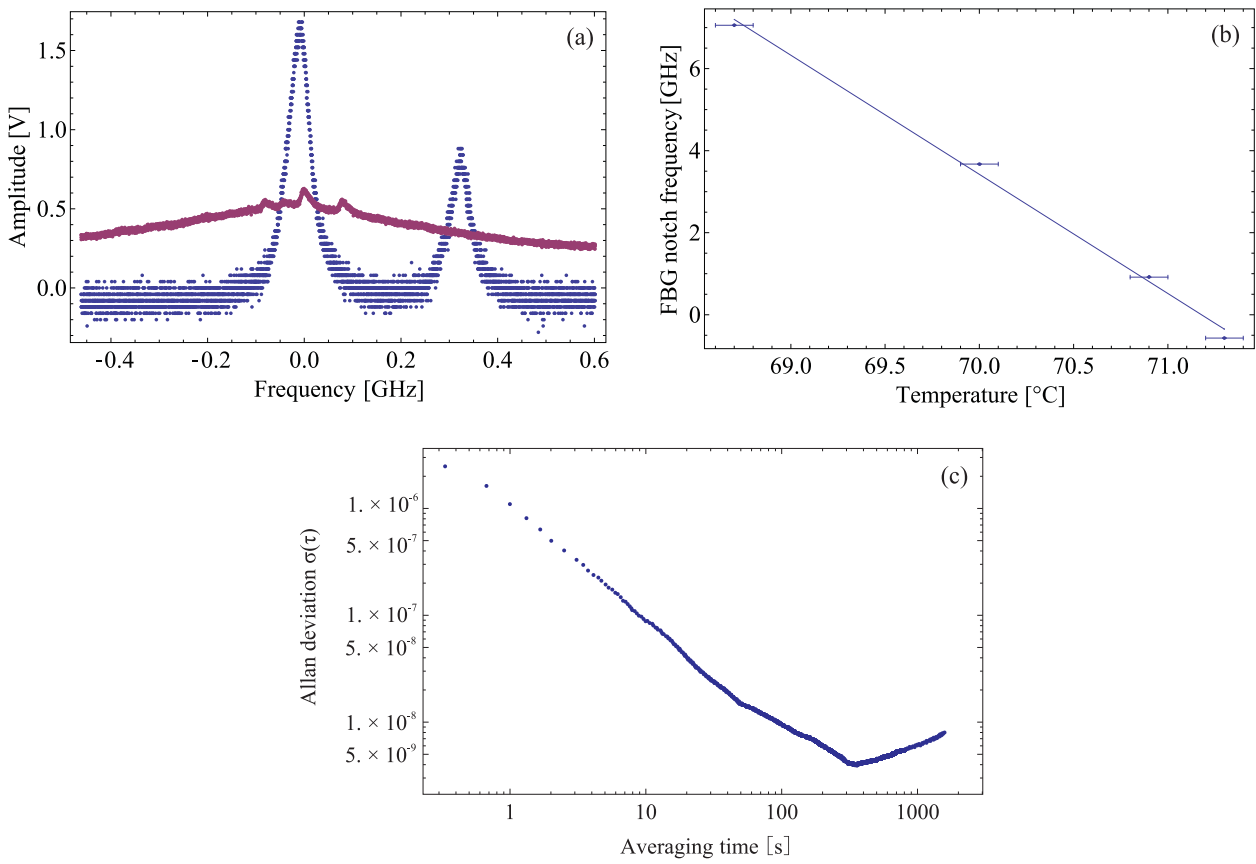

FIG. 9. (a) Calibration of the FBG peak using rubidium resonances. The highest peak in the blue curve, which represents the FBG transmission spectrum was used for calibration. The secondary peak is related to strain and polarizationdependent effects. The red curve represents a saturated absorption spectrum in ${ }^{87} \mathrm{Rb}$. The FWHM of the FBG transmission peak was inferred to be $(70 \pm 1)$ $\mathrm{MHz}$. (b) Tunability of the FBG transmission peak as a function of temperature performed using a Ti:Sapphire laser. (c) ADEV of lock signal recorded with a 780-nm diode laser cavity of type A that was frequency stabilized to the FBG peak. 
were recorded with a Ti:Sapphire laser since it had a larger mode hop-free scan range than the ECDL. The slope of $(-2.90 \pm 0.13)$ $\mathrm{GHz} /$ degree and the range of the temperature controller imply that the lock point can be tuned over a frequency range of approximately $300 \mathrm{GHz}$. In this setup, the resolution of the temperature tuning of the FBG peak allows the lock point to be changed in steps of $3 \mathrm{MHz}$, which is significantly smaller than the typical pulse bandwidth of a LIDAR transmitter $(10 \mathrm{MHz})$. Figure 9 (c) shows the ADEV of the laser frequency locked to the FBG transmission peak. The floor value of the $\operatorname{ADEV}\left(4 \times 10^{-9}\right)$ corresponds to a frequency excursion of $1.2 \mathrm{MHz}$ in $350 \mathrm{~s}$. Since the LIDAR can be operated at repetition rates of $1 \mathrm{MHz}$, it implies that the frequency drifts can remain small compared to the pulse bandwidth until approximately a billion pulses are applied, which constitutes a comfortable measurement window. The ADEV is significantly worse than the data obtained with the lasers locked to atomic resonances (Fig. 5) since the frequency stability of the FBG system is limited by the resolution of the temperature controller. As a consequence, further improvements can be expected with a higher-resolution controller.

\section{CONCLUSIONS}

This paper presents simple adaptations of the well-known IFbased ECDL design that includes the development of an auto-locked controller that can tune and stabilize the laser frequency using various algorithms and a vacuum-sealed cavity that reduces the effects of pressure variations. The long-term performance is characterized at both $633 \mathrm{~nm}$ and $780 \mathrm{~nm}$ using the ADEV of the beat note and of the lock signal. These studies suggest that reducing current noise and cavity pressure are both important for improving the long-term stability of the laser system. Further improvements at lower cavity pressures may be possible since performance limitations appear to be related to outgassing. We incorporate the laser with a state-of-theart commercial gravimeter to demonstrate accurate measurements of gravity that are relevant for natural resource exploration. The auto-locking features of the laser are also potentially useful for realizing the basis of a LIDAR transmitter used in remote sensing to detect trace gases. The versatility of the system has enabled it to be integrated with tapered amplifier waveguides and acousto-optic switches for high-power, pulsed applications. These include industrial magnetometry, ${ }^{42}$ precise determinations of atomic lifetimes, ${ }^{43}$ and atom interferometry with cold atoms.

\section{ACKNOWLEDGMENTS}

We acknowledge helpful discussions with Louis Marmet. We thank Vescent Photonics for the loan of a low-noise current controller. This work was supported by the Canada Foundation for Innovation, the Ontario Innovation Trust, the Ontario Centers of Excellence, the Natural Sciences and Engineering Research Council of Canada, and the York University.

\section{REFERENCES}

${ }^{1}$ C. Affolderbach and G. Mileti, "A compact laser head with high-frequency stability for $\mathrm{Rb}$ atomic clocks and optical instrumentation," Rev. Sci. Instrum. 76, 073108 (2005).
${ }^{2}$ M. T. Hummon, S. Kang, D. G. Bopp, Q. Li, D. A. Westly, S. Kim, C. Fredrick, S. A. Diddams, K. Srinivasan, V. Aksyuk, and J. E. Kitching, "Photonic chip for laser stabilization to an atomic vapor with $10^{-11}$ instability," Optica 5, 443-449 (2018).

${ }^{3}$ D. Akamatsu, Y. Nakajima, H. Inaba, K. Hosaka, M. Yasuda, A. Onae, and F.-L. Hong, "Narrow linewidth laser system realized by linewidth transfer using a fiber-based frequency comb for the magneto-optical trapping of strontium," Opt. Express 20, 16010-16016 (2012).

${ }^{4}$ Y. Cheng, K. Zhang, L.-L. Chen, W.-J. Xu, Q. Luo, M.-K. Zhou, and Z.-K. Hu, "Low-phase noise and high-power laser for Bragg atom interferometer," AIP Adv. 7, 095211 (2017).

${ }^{\mathbf{5}}$ T. M. Niebauer, G. S. Sasagawa, J. E. Faller, R. Hilt, and F. Klopping, "A new generation of absolute gravimeters," Metrologia 32, 159-180 (1995).

${ }^{6}$ D. Naughton, R. Bedington, S. Barraclough, T. Islam, D. Griffin, and B. Smith, Opt. Eng. 58, 1 (2019).

${ }^{7}$ R. Kingsbury, “Optical communications for small satellites," Ph.D. thesis, Massachusetts Institute of Technology, 2015.

${ }^{8}$ A. S. Arnold, J. S. Wilson, and M. G. Boshier, "A simple extended-cavity diode laser,” Rev. Sci. Instrum. 69, 1236-1239 (1998).

${ }^{9}$ M. G. Littman and H. J. Metcalf, "Spectrally narrow pulsed dye laser without beam expander," Appl. Opt. 17, 2224-2227 (1978).

${ }^{10}$ S. Stry, S. Thelen, J. Sacher, D. Halmer, P. Hering, and M. Murtz, "Widely tunable diffraction limited $1000 \mathrm{~mW}$ external cavity diode laser in Littman/Metcalf configuration for cavity ring-down spectroscopy," Appl. Phys. B 85, 365-374 (2006).

${ }^{11}$ T. P. Dinneen, C. D. Wallace, and P. L. Gould, "Narrow linewidth, highly stable, tunable diode laser system,” Opt. Commun. 92, 277-282 (1992).

${ }^{12}$ B. Dahmani, L. Hollberg, and R. Drullinger, "Frequency stabilization of semiconductor lasers by resonant optical feedback," Opt. Lett. 12, 876 (1987).

${ }^{13}$ H. Chang, K. Myneni, D. D. Smith, and H. R. Liaghati-Mobarhan, "Highprecision, accurate optical frequency reference using a Fabry-Perot diode laser," Rev. Sci. Instrum. 88, 063101 (2017).

${ }^{14}$ W. Lewoczko-Adamczyk, C. Pyrlik, J. Hager, S. Schwertfeger, A. Wicht, A. Peters, G. Erbert, and G. Trankle, "Ultra-narrow linewidth DFB-laser with optical feedback from a monolithic confocal Fabry-Perot cavity," Opt. Express 23, 9705-9709 (2015).

${ }^{15}$ Q. Lin, M. A. Van Camp, H. Zhang, B. Jelenkovic, and V. Vuletic, "Longexternal-cavity distributed Bragg reflector laser with subkilohertz intrinsic linewidth," Opt. Lett. 37, 1989-1991 (2012).

${ }^{16}$ Stanford Research Systems, Model PRS10 Rubidium Frequency Standard, 2015 .

${ }^{17}$ B. Bernhardt, T. W. Hansch, and R. Holzwarth, "Implementation and characterization of a stable optical frequency distribution system," Opt. Express 17, 16849-16860 (2009).

${ }^{18}$ C. E. Wieman and L. Hollberg, "Using diode lasers for atomic physics," Rev. Sci. Instrum. 62, 1-20 (1991).

${ }^{19}$ K. G. Libbrecht, R. A. Boyd, P. A. Willems, T. L. Gustavson, and D. K. Kim, "Teaching physics with $670 \mathrm{~nm}$ diode lasers-Construction of stabilized lasers and lithium cells," Am. J. Phys. 63, 729-737 (1995).

${ }^{20}$ L. Ricci, M. Weidemuller, T. Esslinger, A. Hemmerich, C. Zimmermann, V. Vuletic, W. Konig, and T. W. Hansch, "A compact grating-stabilized diode laser system for atomic physics,” Opt. Commun. 117, 541-549 (1995).

${ }^{21}$ X. Baillard, A. Gauguet, S. Bize, P. Lemonde, P. Laurent, A. Clairon, and P. Rosenbusch, "Interference-filter-stabilized external-cavity diode lasers," Opt. Commun. 266, 609-613 (2006).

${ }^{22}$ M. Gilowski, C. Schubert, M. Zaiser, W. Herr, T. Wubbena, T. Wendrich, T. Muller, E. M. Rasel, and W. Ertmer, "Narrow bandwidth interference filterstabilized diode laser systems for the manipulation of neutral atoms," Opt. Commun. 280, 443-447 (2007).

${ }^{23}$ S. D. Saliba, M. Junker, L. D. Turner, and R. E. Scholten, "Mode stability of external cavity diode lasers,” Appl. Opt. 48, 6692-6700 (2009).

${ }^{24}$ A. V. Carr, Y. H. Sechrest, S. R. Waitukaitis, J. D. Perreault, V. P. A. Lonij, and A. D. Cronin, “Cover slip external cavity diode laser," Rev. Sci. Intrum. 78, 106108 (2007). 
${ }^{25}$ E. C. Cook, P. J. Martin, T. L. Brown-Heft, J. C. Garman, and D. A. Steck, "High passive-stability laser-diode design for use in atomic-physics experiments," Rev. Sci. Instrum. 83, 043101 (2012).

${ }^{26}$ F. Kunze, S. Wolf, and G. Rempe, "Measurement of fast frequency fluctuations: Allan variance of a grating-stabilized diode laser," Opt. Commun. 128, 269-274 (1996).

${ }^{27}$ K. Fukuda, M. Tachikawa, and M. Kinoshita, “Allan-variance measurements of diode laser frequency-stabilized with a thin vapor cell,” Appl. Phys. B 77, 823-827 (2003).

${ }^{28}$ B. Barrett, A. Carew, H. C. Beica, A. Vorozcovs, A. Pouliot, and A. Kumarakrishnan, "Prospects for precise measurements with echo atom interferometry," Atoms 4, 19 (2016).

${ }^{29}$ H. C. Beica, A. Carew, A. Vorozcovs, P. Dowling, A. Pouliot, G. Singh, and A. Kumarakrishnan, “An auto-locked diode laser system for precision metrology," Proc. SPIE 10086, 100860W (2017).

${ }^{30}$ H. C. Beica, A. Carew, A. Vorozcovs, P. Dowling, A. Pouliot, B. Barron, and A. Kumarakrishnan, "An auto-locked diode laser system for precision metrology and lidar applications," Proc. SPIE 10191, 101910K (2017).

${ }^{31}$ W. J. Riley, Handbook of Frequency Stability (NIST, 2007), Vol. 1065, pp. 1-123.

${ }^{32}$ H. Talvitie, M. Merimaa, and E. Ikonen, "Frequency stabilization of a diode laser to doppler-free spectrum of molecular iodine at $633 \mathrm{~nm}$," Opt. Commun. 152, 182-188 (1998)

${ }^{33}$ D. A. Steck, Rubidium 85 D Line Data, (revision 2.1.4), December 2010.

${ }^{34}$ K. G. Libbrecht and J. L. Hall, “A low-noise high-speed diode laser current controller,” Rev. Sci. Instrum. 64, 2133-2135 (1993).
${ }^{35}$ K. S. Repasky, J. A. Shaw, J. L. Carlsten, M. D. Obland, L. S. Meng, and D. S. Hoffman, "Diode laser transmitter for water vapor dial measurements," in International Geoscience and Remote Sensing Symposium (IGRASS) (IEEE, 2004), pp. 1947-1950.

${ }^{36}$ A. Dinovitser, M. W. Hamilton, and R. A. Vincent, "Stabilized master laser system for differential absorption lidar," Appl. Opt. 49, 3274-3281 (2010).

${ }^{37}$ J. A. Whiteway, L. Komguem, C. Dickinson, C. Cook, M. Illnicki, J. Seabrook, V. Popovici, T. J. Duck, R. Davy, P. A. Taylor, J. Pathak, D. Fisher, A. I. Carswell, M. Daly, V. Hipkin, A. P. Zent, M. H. Hecht, S. E. Wood, L. K. Tamppari, N. Renno, J. E. Moores, M. T. Lemmon, F. Daerden, and P. H. Smith, "Mars water-ice clouds and precipitation," Science 325, 68-70 (2009).

${ }^{38}$ P. Piironen and E. W. Eloranta, "Demonstration of a high-spectral-resolution lidar based on an iodine absorption filter," Opt. Lett. 19, 234-236 (1994).

${ }^{39}$ C. J. Grund and E. W. Eloranta, "University of Wisconsin high spectral resolution lidar," Opt. Eng. 30, 6-12 (1991).

${ }^{40}$ A. Pouliot, H. C. Beica, A. Carew, A. Vorozcovs, G. Carlse, and A. Kumarakrishnan, "Auto-locking waveguide amplifier system for lidar and magnetometric applications,” Proc. SPIE 10514, 105140S (2018).

${ }^{41}$ G. Spirou, I. Yavin, M. Weel, A. Vorozcovs, P. R. Kumarakrishnan, A. Battle, and R. C. Swanson, "A high-speed-modulated retroreflector for lasers using an acoustooptic modulator,” Can. J. Phys. 81, 625-638 (2003).

${ }^{42}$ A. Pouliot, H. C. Beica, A. Carew, A. Vorozcovs, G. Carlse, B. Barrett, and A. Kumarakrishnan, "Investigations of optical pumping for magnetometry using an auto-locking laser system," Proc. SPIE 10637, 106370A (2018).

${ }^{43}$ H. C. Beica, A. Pouliot, A. Carew, A. Vorozcovs, P. Dowling, G. Carlse, B. Barron, and A. Kumarakrishnan, "Applications of auto-locked laser systems for precision metrology,” Proc. SPIE 74(2A), 65 (2018). 